Even when every conceivable precaution is taken, a single salt is not an adequate basis for the certain decision of an atomic weight. For this reason a parallel investigation on potassium bromide was simultaneously in progress at the Chemical Laboratory of Harvard College. The next communication, describing this other research, must be considered in connection with the work which has just been described. As will be seen, excellent confirmation of the present work is afforded by the work with the bromide.

\title{
Summary.
}

This investigation concerning the quantitative composition of potassium chloride resembled in many respects the recent investigation of Richards and Wells on sodium.

In several details, however, improvements were introduced which effected a considerable saving of time and a perceptible gain in accuracy.

The precautions necessary for the accurate use of the Gooch-Munroe perforated crucible were ascertained; its employment was found to be advantageous.

Platinum weighing bottles with conical ground-platinum stoppers were used instead of boats and glass tubes for weighing the potassium salt.

Occlusion of silver nitrate by the precipitated chloride was diminished by allowing the latter to stand for a long time in a solution containing neither excess of silver nor excess of soluble chloride, and by adding more silver nitrate only after the precipitate had assumed a fairly permanent condition of aggregation.

The nephelometric estimation of small amounts of suspended silver chloride was increased in accuracy by redissolving in ammonia both of the opalescent precipitates to be compared, and reprecipitating, in order to equalize the conditions.

As final results, the outcome of twelve experiments, I00.000 parts of silver were found to correspond to 52.0118 parts of potassium chloride, and 100.000 parts of silver chloride were found to correspond to 69.1073 of this salt.

The corresponding values for the atomic weight of potassium (if silver is assumed to be 107.930 and chlorine 35.473 ) are 39 . I I 34 and 39 . I 145 , in unusually close agreement.

[Contribution from the Chemical Laboratory of Harvard College].

\section{A REVISION OF THE ATOMIC WEIGHT OF POTASSIUM. THE ANAL- YSIS OF POTASSIUM BROMIDE}

By ThEODORE WILLIAM RichaRds aND EDWARD MUELLER. Reeeived February I5, I907.

\section{Introduction}

The foregoing quantitative study of potassium chloride by Arthur Staehler and one of the present authors affords strong evidence that 
the atomic weight of potassium is about $39 . I_{4} 4$, slightly lower than the value based upon the work of Stas. The authors fully appreciated, however, that the investigation of a single compound is not enough to establish a chemical constant so important as this, and, accordingly, the present investigation was prosecuted simultaneously. It was expected that the two investigations might either support one another or else, by affording incompatible results lead to the discovery of a constant error in one or the other, and thus pave the way for further advance in knowledge. As will be seen, the work on the chloride was satisfactorily confirmed by the work on the bromide.

In the present case the careful stucy of potassitum bromicle was particularly necessary, because there already exist two concordant series of experiments upon this substance, performed by the old masters Marignac and Stas, pointing to a ralue in the neigliborhood of 39. I 4, instead of the before-mentioned new value $39.1 \mathrm{I}$. In this case the higher value for potassium is not diminisled, as in the case of the chloride, by an additive correction in the atomic weight of the halogen, because Baxter has shown Stas's estimate for bromine to have been nearly correct.' Hence the discrepancy remains one too serious to be tolerated.

The careful study of this work of Stas and Marignac affords convincing evidence that the potassium bromide used by them for analysis was not sufficiently pure for the purpose. Stas admitted that some of his preparations were not eren wholly soluble in water, and his method of procedure was such that some of them probably contained platinum and hydroxide. Because speculations of this kind concerning work so long past are of but little value, it was clearly necessary to repeat this work with modern care; and the following pages recount the details of the repetition.

In common with others of the same type, the investigation easily resolves itself into several sections and will be discussed under the following heads: The preparation of materials; the drying and weighing of potassinm bromide; the determination of the ratio of silver bromide to potassinm bromide; the deternination of the ratio of silver to potassium bromide; and the discussion of the results. Of the laboratory tasks the preparation of the materials mas by far the most laborious and puzzling, while the analytical work was comparatively simpie.

\section{The Preparation of Materials}

The Source of the Potassizm. - The task of preparing pure potassinin bromicle, simple as it may appear, is by no means an easy one. In riew

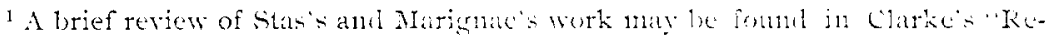
calculations" (IS97), p. 47. Inxter" work is to he fomm in Pt. An. Acal, 42, 201 .

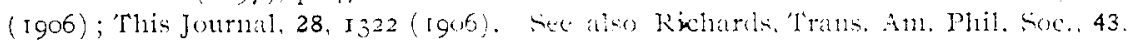
I 6 (1904. 
of our experience, it seems probable that neither Marignac nor Stas ever prepared samples pure enough to correspoud with the other precautions which they took. As in the case of sodium bromide ${ }^{1}$ the salt itself when once made can not be effectively purified. In this respect it differs widely from the chloride. We found after due trial that the potassium and bromine must be purified separately in such forms as to introduce no foreign matter. Accordingly, the two substances, already adequately purified, were caused afterwards to yield potassium bromide; and this salt, when recrystallized, gave the final substance for analysis. The purification of the potassium materials will be discussed first.

It was necessary to provide potassium material which should be free from the suspicion of introducing with the metal desired, anv impurity either basic or acid. Potassium nitrate, which had served so well in the case of the chloride, was not suitable for the present purpose, because the destruction of the nitric acid involves too great a sacrifice of laboriously prepared hydrobromic acid. After due consideration, potassium oxalate was tentatively chosen. It was necessary to prove that this salt can be easily and certainly purified by recrystallization, and especially to show that the other alkali metals can thus be separated. The following tests accomplished this proof.

A saturated solution of potassium oxalate containing a purposely added admixture of to per cent. of sodium oxalate was crystallized and the crystals drained centrifugally. By the flame test, a pronounced difference was noticeable between the amount of sodium present in the mother liquor and that in the crystals. A second recrystallization and whirling was still more satisfactory; the crystals gave no sodium test to the eye, while the mother liquors gave a distinct yellow. This indicates that sodium has a decided tendency to pass into the mother liquors, and hence can be separated by fractional crystallization.

Again, I per cent. of lithium salt was added to potassium oxalate with similar outcome. Rubidium and cesium were tested likewise. A saturated solution of potassium oxalate and a salt of each of these metals was made of such strength that the rubidium or cesium could easily be found by means of the spectroscope. Each solution was evaporated until saturated, and cooled; and the crystals were whirled. The mother liquor gave a test for the impurity, the crystals gave none.

No other oxalates were seriously to be feared, because the insoluble ones would appear at once and demand filtration, and soluble double oxalates could hardly be isomorphous, and would therefore tend into the mother liquors. Traces of calcium oxalate in fact appeared in the former fashion, and traces of iron in the latter.

${ }^{1}$ Richards and Wells, Pr. Am., Acarl, 41, 435 (1906); Z. physik. Chem., 56, 348 ( 1906$)$. 
In view of these satisfactory results, it was evidently only necessary to crystallize the potassium oxalate often enough to make the separation complete; and since potassium oxalate has a fairly convenient change in solubility with the temperature, the yield after a considerable number of crystallizations is good, especially if pains are taken to carry out the process systematically.

Two very pure samples of oxalate were made, from tmo different sources. As one source of material, a commercial sample prepared by Merck was used. It had been labeled "Potassium oxalate, neutral, highest purity," and contained as a matter of fact only the traces of calcium and iron already referred to, but no lead or other discoverable inpurity. A large quantity of a solution of this salt was filtered hot into a platinum dish, where it was five times systematically recrystallized, the crystals being centrifugally whirled each time.

Careful scrutiny in the spectroscope revealed in the product no other spectroscopic lines than those of potassium, and careful qualitative tests proved the absence of lead and iron. The salt dissolved without a trace of residue in water, and was quite pure enough to serve as a starting point for further operations, to be described under the heading "Potassium bromide."

A second sample of potassium material was obtained from Merck in the form of the hydroxide, being marked "Potassinm Hydrate, Chemically Pure Reagent ; Conforms to the standards of Dr. Krauch." It was in fact very pure, giving a perfectly clear solution both before and after neutralization. It was neutralized with a specimen of oxalic acid which had been carefully purified by Mr. G. E. Behr, Jr, for other work. This acid contained no halogens, and left no residue on volatilization in platinum. Slightly more than the equivalent quantity of acid was added, and the oxalate obtained was therefore slightly acid. As will be shown, the acidity was later the cause of much inconvenience, and had better have been aroided. The oxalate was four times systematically recrystallized in platinum with centrifugal draining each time. The final product, like the previous one, gave no tests for impurities except a slight excess of acid; it was used as the source of potassitim for one preparation of potassium bromide.

Bromine.-Although the methods previously used at Harvard had afforded a satisfactory yield of very pure bromine, ${ }^{1}$ it was desired to add to the knowledge on the subject by testing another method. Through the generosity of the Mallinckrodt Chemical Works of St. Louis, Missouri, and also of the Dow Chemical Works of Midland, Michigan, a large quantity of potassium bromate was placed at our disposal. Repeated.

${ }^{1}$ See Pr. Am. Phil. Soc., 43, IIg (1904), for references and Sias, Mémi. Acad. Belg., 43, II, $38($ ISS2). 
crystallization very kindly carried out for us on a large scale by these firms, had been the method used in its purification.

This material, which served as our starting-point, was already very pure, a fact which was indicated by three syntheses of silver bromide from purest silver and potassium bromide obtained by decomposing the Mallinckrodt bromate. These syntheses, carried out with all necessary precautions gave, respectively, 57.44 I, 57.440, 57.44 I as the percentage of silver in silver bromide. According to Baxter the true amount is $57.445 .{ }^{1}$ The slight difference indicates a trace of iodine rather than chlorine; but this impurity can be easily expelled, while chlorine is far more troublesone.

It having been demonstrated that recrystallization is an effective means of purifying the salt from chlorate and chloride, a large quantity of potassium bromate was three times recrystallized in porcelain, the crystals being centrifugally whirled each time and all the mother liquors discarded. This gave an exceedingly pure bromate, much purer than Stas could have obtained ${ }^{2}$ because the centrifugal draining makes so great a difference in the rate of purification. ${ }^{3}$

A large quantity of the salt was decomposed into the bromide in a platinum dish by the heat of an alcohol lamp. The potassium bromate in decomposing attacked the platinum, and the mass of bromide was slightly brownish in color, but this caused no inconvenience in the present case, as the bromine was to be subsequently distilled. In order to liberate the halogen, the bromide was treated in strong solution with less than the calculated quantity of bromate and an excess of pure sulphuric acid. The acid was added drop by drop to the mixed solution of salts in an apparatus made entirely of Jena glass, which was kept surrounded by ice-water. The bromine which volatilized on account of the heat of the reaction was caught in a condenser under water; to this was added the remaining bromine, which had been separated from the supernatant solution by means of a separating funnel.

The bromine thus obtained was twice distilled by steam heat into water in a Jena glass condenser, packed in ice. It was kept in a well-seasoned bottle with double ground-glass stoppers.

The product was certainly free from non-volatile materials, and could have contained only iodine and perhaps a trace of sulphuric acid as impurity. It will be seen that these were eliminated in the subsequent work. This was the only sample of bromine prepared; it was used in all the preparations of bromide, as the quantitative results indicated that it was very pure.

${ }^{1}$ Baxter, This Journal 28, I 332 (1906). See also Richards, Trans. Am. Phil. Soc., 43, IIg (1904).

2 Untersuchungen, 160.

${ }^{3}$ Richards, This Journal, 27, 104 ( 1905 ). 
Pstassizm Bromide. - The problem of obtaining potassium bromide from the oxalate and bromine had been carefully considered before these substances had been prepared. Only two methods recommended themselves for the present purpose, atthough the bromide can be obtained from these two materials by a variety of reactions, which would ordinarily be acceptable. Here, however, simplicity, completeness, and theoretical correctness must all be fulfilled; that the methods adopted really possessed these advantages in their faror must be adnitted after careful perusal of the following cescription of the details.

In some preliminary experiments it was shown that by adding an excess of bromine to the oxalate and evaporating off the excess, a very pure bromicie quite free from oxalate is obtained. Accordingly, a quantity of the first sample of five times crystallized potassium oxalate was treated with an excess of bromine, the bromine being added in small quantities from a dropping funnel. The solution was held in a quartz dish, which was carefully hooded to prevent entrance of dust. When the action had ceased, the solution was heated on a steam-bath mintil any traces of iodine and all excess of bromine had been expelled. The solution was found to be entirely free from oxalic acid upon testing with calcium nitrate in a solution faintly acid with nitric acid. Although it was wholly colorless while in the quartz dish, after being concentrated in platinum it dereloped a very faint yellow tinge. Upon recrystallizing three times this color was finally concentrated in the extreme mother liquor, and was found to be due to trace of platinum; no iron was present. Even the first crystals were perfectly white, the second and third mother liquors also showed no trace of color, and only the first of the mother liquors gave a test for platinum.

The material thus prepared, after being three times recrystallized in platinum with centrifugal draining, was called sanple $I$, and served for analyses I to II.

The second sample of potassium bromide was prepared from the second sample of four times recrystallized potassium oxalate. The method was the same as with the previous sample. An excess of bromine was added to the oxalate in quart $z$; when action ceased, the bromine was driven off by heating on a stean-bath. However, on testing for oxalate, a perceptible quantity was found still undecomposed. This was unexpected, in view of the fact that the first preparation of bromide had given no trouble in this respect. The difficulty was traced to the presence of a smail percentage of acid salt in the oxalate; the presenee of acid lias an intubitory effect on the reaction of bromine on the oxalate ion. Obviously, the simplest remedy would have been to adi pure potassium hydroxide; but this was not added because at that moment there was none at hand. Ioreover, because the reaction was being performed in quartz, it was 
thought best not to use a caustic alkali for fear that the dish might be slightly attacked. The addition of a small amount of very pure recrystallized bromate to this slightly acid solution served better, because its use was unattended with danger. Its employment was entirely effective; the ionized hydrogen was removed, and after adding more bromine and heating, a solution of bromide was obtained which contained no trace of oxalate.

This specimen was recrystallized entirely in quartz, in order to prevent a recurrence of the trouble experienced with sample I, as well as to see if the variation of conditions affected the atomic weight. T'wo crystallizations were made with centrifugal draining, and every precaution; and there was obtained finally a pure, colorless product with which were made two analyses, Nos. I 2 and I3. That a uniformly pure material had been made is shown by the close agreement of these two analyses with the mean of all.

As a further precaution against accidental error, it was thought best to develop a method essentially different from that just discussed, and to prepare therewith a third specimen of bromide. In brief, this method consisted in the separate preparation of pure ammonium bromide and potassium hydroxide, and the production of potassium bromide by the evaporation of the mixed solutions of these substances. Thus can be obtained a salt contaminated only with excess of volatile ammonium bromide.

The preparation of pure ammonium bromide was very easy. Ammonia essentially free from carbon compounds was redistilled into water in a platinum dish. Into this was dropped the purest bromine, which fell through the liquid into a small porcelain crucible resting on the bottom of the dish. In this way the bromine itself could not come into contact with platinum and attack it. The reaction took place very rapidly, yielding ammonium bromide which could have contained no non-volatile ingredients, because all its constituents had just been distilled.

The preparation of pure potassium hydroxide was less easy to devise, because more original; but the execution was almost as easy. The problem was solved by the use of an electrolytic process which will be described in greater detail elsewhere. In brief, a saturated solution of potassium oxalate (sample I) was electrolyzed between a pure mercury cathode and a platinum anode in a porcelain dish cooled with ice, the current from four storage cells being used. When the amalgam became solid, the current was stopped, the solution decanted, and the amalgam washed, being triturated with an agate pestle under water, till vo test for oxalate could be obtained. The pure amalgam was transferred to a platinum dish, covered with water, and made the anode of a dense current until only a little of the potassium amalgam remained undecomposed. 
Thus pure potassium hydroxide was formed. This solution was poured into the ammonium bromide solution, the mixture was evaporated and crystallized, and the salt thus obtained used for analyses 14, I 5 and I6.

The bromide thus obtained must have been very pure. During the electrolysis, the high concentration of the potassium oxalate would tend to allow the deposition of potassium only." At any rate, metals less easy to deionize could hardly have been set free. During the later decomposition of water by the amalgam, all metals with less tendency to ionize would have remained in the mercury, because some of the amalgam was left undecomposed. Any trace of iodine which the bromine may have contained must have been driven off by the fusion just before analysis. this fusion being prolonged in order to expel ammonium bromide. The results of the analyses of this sample indicate its essential identity with the two preceding preparations. Since there was no known source of impurity in any one of the preparations, this was not surprising.

Although our consistent employment of insoluble vessels rendered the presence of colloidal silica unlilety, an effort was made to test for this impurity of sample II by volatilizing 3 grams of it in a stream of nitrogen. Unfortunately, the high temperature of the electric furnace caused the platinum boat containing the salt to weld to the foil which surrounded it in the tube, so that no certain quantitative evidence could be obtained. Two ninute black spots were visible in the boat; nothing else could be seen upon very careful scrutiny. If there were any silica present, the amount must have been too small to have had any appreciable influence on the final atomic weights. Tine and material were lacking, hence the experiment was not repeated.

Silicr...-The essential details of the preparation of pure silver, as developed by Richards and Wells, were followed in detail, and the reader is referred to their paper, as well as to the preceding paper of Richards and Staehler, for the particulars. Two samples of silver were made; they were found to be identical in quantitative behavior.

The source of the material used for the first preparation was the pure precipitated silver chloride remaining from the work of Richards and Wells on sodium. It was reduced to metallic silver with invert sugar in a strong solution of sodium hydroxide. The silver thus obtained was washed free from soluble matter and dissolved in nitric acid; the silver nitrate after crystallization and whirling was reduced with ammonium formate, yielding a beautiful crystalline mass of metal.

This pure silver, after very thorough washing, was fused on lime by means of a blast lamp rhose tip had been carefully cleaned. The buttons which were thus obtained were cooled in the reducing flame, washed free

1 See Bunge, Ber. 9, 78 ( 1876 ).

"Berthelot. Ann. chim. phys. [5] 18, 43.3, (1879). 
from lime, scrubbed with cleaned sea-sand, etched with strong nitric acid, and washed with the purest water, to serve as anodes in the electrolytic purification which was the next step. The eloctrolysis was carried on as already described in previous similar Harvard researches, and every step of the subsequent work may befound in detail in the preceding papers on sodium and potassium chloride. ${ }^{1}$ The metal was finally fused as usual on a boat of the purest lime in an atmosphere of hydrogen under a tension of $50 \mathrm{~mm}$. The larger pieces, after etching with nitric acid, were cut into smaller ones of convenient size with a clean cold chisel. They were again etched in order to dissolve all traces of iron, ${ }^{2}$ scrubbed with clean sand, washed with a jet of water, and etched yet again with acid, paying particular attention to the cut edges. Finally the pieces were dried over an alcohol lamp and kept in a desiccator over potassium hydroxide for use.

This silver was used both in most of the titrations against potassium bromide and as the source of silver nitrate used in the precipitation of silver bromide to be weighed.

A second sample of silver, prepared from somewhat less pure initial material, was put through a more elaborate and exhaustive round of purification which need not be detailed. The preparation included five crystallizations as nitrate and two successive precipitations as metal by formate. This silver was fused into buttons of a size suitable for immediate use without cutting. They were cleaned by etching and washing, dried and preserved free from impurity as usual, and were used in analyses 7 and 9 .

Nitric Acid.-For preliminary work, ordinary "chemically pure" nitric acid was distilled once, using a platinum condenser and Jena glass receiver, the first third of the acid being discarded. For the final analyses this redistilled product, already very pure, was once again distilled, as before, the first third being rejected. This distillate gave no test with the nephelometer, after dilution.

Water.-As usual in such work, all the water used had been carefully purified by double distillation, once from a fairly strong solution of alkaline potassium permanganate, and then alone. The distillate was caught in Jena glass receiving flasks which were provided with special adapters to prevent the access of dust. The first and last fractions were always rejected, only the middle portion being used. The connection of the Jena glass boiling flask with the pure tin condenser was made without rubber

${ }^{1}$ See Pr. Am. Acad., 38, 450 (1903) ; and Richards and Welts, This Journal, 27, 473 (rgo5); Richards and Staehler, preceding paper.

${ }^{2}$ Richards and Archibald, Pr. Am. Acad., 38, 450 (I903); Baxter, This Journal, 28, 1329 ( I906). 
or cork.' Such water gave every evidence of sufficient purity, as has been frequently pointed out.

The Laboratory.-A very essential precaution in every stage of this work was to effectually exclude hydrochloric acid. Accordingly, all the preparations of the bromide were carried out in a private laboratory, used especially and only for this nork, and wholly free from the interference caused by large classes or other researches. Great care was taken not to allow any hydrochloric acid in the room; indeed, not a drop was used there during the entire research. A separate ventilating fan prevented fumes from other laboratories from interfering with the work.

Ctensils.-As usual, great care was taken to avoid the use of any vessels which under the given conditions might be attacked and thus pollute the substance in hand. When glass and porcelain were not harmful, as in the early stages of the preparation of silver, sucln receptacles were employed; but in the purification of the substance for analysis, platinum and quartz were always used.

\section{The Drying and Weighing of Potassium Bromide.}

For reasonsalready mentioned in the paper on potassium chloride, the salt under investigation must be fused before weighing. Unfortunately, potassium bromide when fused in air in a platinum ressel attacks the platinum to a considerable extent. Although it is shown later that this action does not seriously affect the weight of silver needed to precipitate the bromine in the salt, it was nevertheless desirable to aroid all such irregularities. No such action is observed when the fusion takes place in an atmosphere of pure dry nitrogen. Accordingly, in all work to be recounted, the potassium bromide was protected in this way during its fusion in a well-seasoned platinum boat, and was finally, by means of the Harvard "bottling apparatus," shut up in a tight weighing bottle in an atmosphere of pure dry air. The details have already been adequately given in several places, especially in the account of the recent work on the atonic weight of cesium. ${ }^{2}$ The only difference in the present procedure was demanded by the high fusing-point of potassium bromide $\left(750^{\circ}\right)$; on account of this, a porcelain ignition-tube was substituted for one of glass, as in the case of calcium chloride. ${ }^{3}$

Even with all possible care however, the platinum boat was distinctly attacked, almost always losing in weight during the fusion. In two of the cases-analyses I and I4-where the potassium bromide was fused for some time in the boat, the corrosive action was very considerable, causing losses of weight of 0.0007 and 0.0014 gram, respectively. One would be been frequently pointed out.

'Richards, Pr. Am. Acad., 30, 380 (IS94).

'Richards and Archibald, Pr. Am. Acad, 38, 45 I (1903); Z. anorg. Chem., 34, 362 (1903).

Richards, This Joumal, 24, 374 (J902). 
disposed to reject these determinations entirely, except for the fact that their average result, $\frac{\mathrm{KBr}}{\mathrm{Ag}}=1.10317$, is almost exactly identical with that of the average of results 5 and 6 , in which the boat was scarcely attacked at all namely, $\frac{\mathrm{KBr}}{\mathrm{Ag}}=1.0318$. Instead of being rejected, these results are therefore retained and used as evidence that even a considerable amount of dissolved platinum has no perceptible effect on the reight of silver precipitated by the salt. In a majority of other cases, the change in weight of the boat during the fusion was less than 0.0 cor gram. The average loss in analyses IO, II, I2, and I6, in which the silver bromide was weighed, was less than 0.00009 gram; hence even if platinum had been present in the potassium bromide in a state of fine division it could not have exerted any essential effect upon the result of this series of analyses.

As a matter of fact, the potassium bromide always gave a perfectly clear aqueous solution except once, namely, in analysis I4. In this case several small flakes of platinum were noticed in the salt both before and after the salt was dissolved in water.

The solutions were always perfectly neutral to phenolphthalein, a fact which is adequate proof of the retention of all the bromine during fusion, and aiso of the absence of oxalate from the potassium bromide crystals.

The third sample of potassium bromide had contained ammonium bromide, which was expelled during the fusion. During the expulsion of this salt, the boat was placed near the exit end of the porcelain tube, and there gradually heated to redness in a current of nitrogen. During the entire operation the middle portion of the porcelain tube waskept red-hot in order to prevent backward diffusion of the ammonium bromide, which by condensing might later cause a contamination of the potassium bromide. All the ammonium bromide having been sublimed, the boat was pushed into the middle of the tube, and the potassium bromide fused as described above. There being a possibility that even at the fusing-point of potassium bromide some ammonium bromide might still be retained, another portion of the same third sample of bromide was fused under approximately the same conditions as in the analyses. The solution of the bromide thus obtained was submitted to the Nessler test, being compared with a tube of pure water containing the same amount of reagent. No color was visible in either tube, hence no appreciable amount of ammonium bromide remained to contaminate the potassium salt.

The salt thus prepared for analysis was weighed in its glass-stoppered weighing-bottle by substitution, using as the substituting tare a precisely similar weighing-bottle. Successive weighings of the same specimen were always practically identical. The balance and weights were similar 
to those used with sodium and potassium chlorides, and all the precautions were the same. At each weighing the heights of the barometer, the temperature of the balance-room, and the humidity as indicated by a hygrometer were recorded. The density of the displaced air could thus be determined for each case. The variations from the average were, however, not enough to affect essentially any of the results, as weighings were not made under any abnormal atmospheric conditions. The weights were of course standardized by the usual Harvard method.

The specific gravity of potassium bromide must be known to within five one-hundredths of a unit in order to correct the weighings to the vacuum standard with sufficient accuracy. Its determination ${ }^{1}$ has been undertaken with a variety of methods, with a variety of results, ranging from 2.20 to 2.756 . The more recent results all tend toward the highest value, found by Krichmeyer" (2.756). This appeared to be the most probable, because the usual errors cause low results; but as this determination was made with only a single crystal, and as the salt weighed in the present research had been fused, it was thought best to investigate further. Accordingly, a pure sample of potassium bromide was further purified by being three times recrystallized with centrifugal draining, when it gave no flame test for sodium." This product was fused in platinum and coarsely pulverized; its specific gravity was determined in an Ostwald pycnometer, modified for use with solids, using toluol as the fluid to be displaced. The tolnol had been redistilled and possessed a density 0.8608 at $25^{\circ}$, referred to water at $4^{\circ} ; 8.2568$ grams of potassium bromide displaced $2.60\left(9 \mathrm{grams}\right.$ of the toluol at $25^{\circ}$, a result which indicates a density of 2.73. Time was lacking for a repetition of the experiment, but this preliminary result was enough to show that the fused salt is essentially in the same state as the crystallized salt. The difference between the two values is too small to affect the vacuum correction; the average value 2.74 was used.

\section{The Precipitation and Weighing of Silver Bromide}

In order to determine the weight of silver bromide obtainable from the bromine in potassium bromide, the latter salt was precipitated by a very slight excess of silver nitrate, both substances being dissolved in large quantities of the purest water. The method differed but slightly from that which had been used recently in so many other cases of the same kind. The silver nitrate was prepared from a weighed amount of pure silver, but no attempt was made in this series to determine the exact

${ }^{1}$ For the literature see LandoIt u. Börnstein (Aeyerhoffer). PhysikalischChemische Tabellen (Ig05), and Clarke, Constants of Nature (part r). Macmillan (I888).

"Krichmeyer, Z. physik. Chem., 21, SI (1S96)

"See Krichmeyer's experience, loc. cit. 
amount of silver required, as it was desired not to complicate the process before weighing the silver bromide. Care was taken to have an excess, but only a slight excess. The silver was dissolved in nitric acid with all the usual precautions, and the precipitation was carried out in orange-red light in the dark-room devoted to accurate work of this kind. The potassium bromide had been dissolved out of the boat by digestion in a large platinum dish, and been transterred to a 2 liter glass-stoppered Jena Erlenmeyer flask with careful rinsing, and every precaution to prevent any gain or loss. After being well shaken, the flask containing the silver bromide was allowed to stand until the mother liquor had become clear. The solution was filtered through a platinum Gooch-Munroe crucible, and the precipitate was washed by decantation, first with an extremely dilute acid solution of silver nitrate, and finally with exceedingly dilute nitric acid to prevent colloidal solution. With this latter faintly acid liquid the precipitate was transferred to the crucible. The silver bromide was dried at least twelve hours in the filtering crucible at $130^{\circ}$ in an electric oven, ${ }^{2}$ and weighed. It was then fused in a porcelain crucible, in a furnace where it was effectively protected from the flame gases in order to determine the trace of moisture always retained. The washwaters and the solution obtained on neutralizing the ammoniacal rinsings of the flask gave no indication of the presence of bromine on careful testing in the nephelometer.

In using the Gooch-Munroe crucible, all the precautions pointed out in the preceding paper were carefully heeded. A perforated plate was always placed on top of the friable sponge to prevent rupture. After each determination any bromide clinging to it was leached out with potassium cyanide which was then washed away with nitric acid, and finally very thoroughly with water. The solvent action of the cyanide occasionally loosened portions of the spongy film, but there was no difficulty in repairing the injury. Upon drying in the electric oven the crucible was ready for another analysis.

In addition to the loss of weight on fusion, two other very small and somewhat uncertain corrections were applied to the weight of the silver bromide, but because they were of about the same magnitude and of opposite sign, their effect was practically negligible. These were a correction for platinum corroded from the boat during the fusion of potassium bromide and a correction for silver bromide dissolved in the water used for washing. Because these corrections have not been considered in most work of this kind, a word about them may not be amiss.

In the series under discussion the average loss of the boat in each determination was less than 0.00009 gram. All of this trace of platinum may have been present as invisible dust in the solution, and thus may

${ }^{1}$ Richards, An. Ch. J., 22, 45 (1899). 
have been weighed with the silver bromide, or all of it may have been in a soluble form and may have remaned in solution. Because of this uncertainty a compronise was made, and half the loss of the weight of the boat (in the mean 0.00004 gram) was subtracted from each reight of silver bronide. As the arerage total weight of silver hromide was orer 4 grams, this compromise could not hare introduced an error as great as I part in 100,000 in either direction.

According to Stas, silver bromide is wholly insoluble in water, but recent experiments slow that in the flocculent form it is unquestionably soluble to a slight extent." As in the case of the chloride, its solubility. Chem.. 12, 2.34 (1893); also Kichards, P'r. Am. Acad., 30, 385 (1894).

is greatly diminished by the addition of an excess of either precipitant; but whether or not any is dissolved by a very dilute acid solution of silver nitrate, such as that used in rashing, it is practically impossible to discover. It is not unlikely, however, that about as much was dissolved by this solution as by the dilute hydrobromic acid used hy Baxter in his admirable work on the atomic weight of bromine:" and in this solution it is easy to find the amount of dissolved substance. Baxter found as a matter of fact, in his last seven most exact syutheses an average of 0.00004 gram of silver bromide in each of the wash-waters-a figure which was added to each of our neights of silver bromide, because the other circumstances of the analysis were similar in the two cases. On the average, this correction exactly eliminates the other; and except for the sake of completeness, they might both have been wholly neglected.

Of course all the weighings were corrected to the racuum standard, by adding $0.00004 \mathrm{I}$ gram to every apparent gram of silver bromide and 0.00029 gram to every apparent gram of potassium bromide, as calculated from the figures $6.473,2.74$, and 8.30 , for the densities of silver bromide, "potassium bromide, and the brass weights respectively. All the determinations made are given in the table below.

\begin{tabular}{|c|c|c|c|c|}
\hline $\begin{array}{l}\text { Experiment } \\
\text { number }\end{array}$ & $\begin{array}{l}\text { Weight of } \\
\text { kbrinvactum } \\
\text { grams }\end{array}$ & $\begin{array}{l}\text { Corrected weight } \\
\text { of AgBritl } \\
\text { vac111m } \\
\text { grams }\end{array}$ & $\begin{array}{l}\text { Parts of } \mathrm{KBr} \\
\text { for doo.ooparts } \\
\text { of } \mathrm{AgBr}\end{array}$ & $\begin{array}{l}\text { Atomic Weight } \\
\text { of Potassilm } \\
\text { 135 }=79.95 .3\end{array}$ \\
\hline Io & 2.19027 & 3.45617 & 63.3728 & 39.114 \\
\hline I I & 4.19705 & 6.62285 & $63.3 i^{23}$ & 39.113 \\
\hline 12 & 2.06723 & 3.26206 & 63.3719 & 39.112 \\
\hline 16 & $2.5 \$ 494$ & $4.0-889$ & 63.3736 & 39.115 \\
\hline Total average & I $L .0395$ & 17.41997 & 63.3727 & 39.1135 \\
\hline
\end{tabular}

The extreme deviation from the mean corresponds to an error of weigh-

${ }^{1}$ Stas, Oeuvres, I, 89.

2 Böttger, Z. physik. Clem., 46, 602 (1903); Kohlrausch und Rose, Z. physik.

"Baxter, This Journal, 28, J322 (1906).

' Baxter and Hines, A11. Ch. J., 31, 220 (I904). 
ing the potassium bromide of 0.00004 gram, a reasonable quantity. The "probable error" indicates that there is but little chance that the atomic weight of potassium is much below 39.1 I 3 or much above $39 . I_{4}$, if constant chemical errors were successfully excluded. Stas found in a single experiment the number 63.383 instead of $63.373 .^{1}$

With unlimited time more determinations might well have been made, but the agreement of these four results is so gcod that further repetition seemed to be not very urgent.

\section{The Determination of the Silver Needed for Precipitation}

The silver titration method of Gay-Lussac, as developed by Pelouze, Mulder, Stas, and more especially by the recent work at Harvard, yields very consistent and accurate results, if the proper conditions are carefully observed. The reading of the end-point is rendered easy by the use of the nephelometer. ${ }^{2}$ In the case of the somewhat soluble chloride, various precautions must be strictly heeded; but with silver bromide, which is almost insoluble, the matter is a simpler one, and a very slight excess of either bromide or silver can be easily determined.

The method used in the present case is easily inferred from previous Harvard work of the same kind. From the weight of a piece of the purest silver, the equivalent amount of potassium bromide was calculated; slightly more than this amount of substance was then fused as before in a platinum-iridium boat placed in the porcelain tube of the bottling apparatus. From the weight of fused bromide, the equivalent quantity of silver was calculated; the greater part of the difference between this calculated weight and that of the original piece of silver was added in the shape of pure silver wire, ${ }^{3}$ and any final difference of 0.1 or $0.2 \mathrm{mg}$. was made up with a dilute solution of silver nitrate. The silver was dissolved in nitric acid and the nitrous acid expelled as usual, and the solution was then diluted to about tenth normal.

To the dilute bromide solution, with continual agitation was added this dilute silver solution; the total volume including the water used in rinsing usually amounted to about r.5 liters. After shaking steadily for I5 minutes, and occasionally for a day, the mixture was allowed to settle during a nother day. When clear above, about 0.05 liter of the aqueous solution was withdrawn and tested in the nephelometer, one tube being treated with an excess of bromide, the other with an excess of silver nitrate. If any difference in the opalescence was noticeable after due time had been allowed for the very faint clouds to attain their maxima, the slight deficiency was made up in the

1 Stas, Untersuchungen (Trans. Aronstein 1867) page 340.

${ }^{2}$ Richards and Wells, Am. Ch. J., 31, 235 (1904).

${ }^{8}$ Richards and Parker, Pr. Am. Acad.. 32, 60, (I8c6.) 
flask by means of solutions containing approximately I milligram of silver nitrate or of potassium bromicle per milliliter. These additions were continued till equality in the opalescence was attained. From the sum of the original weights and subsequent additions, the total amounts of bromide and silver were obtained; from these, reduced to vacuum, the ratio was calculated. All the analyses which were performed are given in the table, excepting No. 2, which was rejected for just cause before it was finished. Vacuum corrections of +0.00029 for every gram of potassium bromide and - 0.00003 for every apparent gram of silver were applied. Most of these experiments were made before those given in the preceding table, a fact which may account for the slightly less satisfactory agreement of the individual results. Because the deviations could not be traced to any definite cause of disturbance, they must be ascribed to accident.

THE RATIO OF POTASSICA BROMIDE TO SILVER

\begin{tabular}{|c|c|c|c|c|}
\hline $\begin{array}{l}\text { Experiment } \\
\text { Nunber }\end{array}$ & $\begin{array}{l}\text { Weight of } \mathrm{KBr} \\
\text { in vacumm } \\
\text { grams. }\end{array}$ & $\begin{array}{l}\text { Weight of Silver } \\
\text { invaculum } \\
\text { grams. }\end{array}$ & $\begin{array}{l}\text { parts of } \mathrm{KHr} \\
\text { corresponding to } \\
100.00 \text { parts } \mathrm{Ag}\end{array}$ & $\begin{array}{l}\text { Atomic Weight } \\
\text { of Fotassium } \\
\text { if } \mathrm{Br}=79.953\end{array}$ \\
\hline I & 4.33730 & $3.9316 t$ & I $10,31 \mathrm{~S}$ & 39.113 \\
\hline 3 & 4.18763 & $3 \cdot 79.587$ & 110.320 & 39.115 \\
\hline 4 & 4.15849 & 3.76943 & 110.321 & 39.116 \\
\hline 5 & 3.67867 & 3.33450 & 110.321 & 39.116 \\
\hline 6 & 3.60484 & 3.26776 & 110.315 & 39.110 \\
\hline 7 & 4.78120 & 4.33387 & 110.322 & 39.IIS \\
\hline 8 & 5.67997 & $5.1+860$ & I 10.321 & 39. II 6 \\
\hline 9 & 6.41587 & $5.8157 \mathrm{I}$ & 110.320 & 39.115 \\
\hline I3 & 2.88134 & $2.6118 t$ & 110.318 & 39. II 3 \\
\hline I4 & $3.6+383$ & 3.30309 & I10. 316 & 39. III \\
\hline I 5 & 3.12757 & 2.83504 & 110.319 & 39.113 \\
\hline & & & 110.3190 & ${ }^{1} 39$. I I 43 \\
\hline obable erro & & & 0.0004 & 0.0004 \\
\hline
\end{tabular}

The "probable error" is as small as before because of the greater number of determinations; and the mean deviation from the average value is only one in the last decimal place. It will be observed that these results point to the limits 39 . I I 3 and 39 . I 5 as the extreme values between which the atomic weight of potassium must fall, in essential agreement witb the previous results.

Marignac's seven experiments on this ratio give values ranging from I 0.303 to I 10.369 , while Stas's fourteen results ranged from 1 0.332 to II $0.36 \mathrm{I}$.

Obviously the results furnish a means of calculating the atomic weight of bromine, when taken in connection with the foregoing series, entirely

${ }_{3}$ The average 39.1443 is calculated from the arerage 110.3150, not from the average of the individual values of the atomic weights in the column above. The difference is of course very slight. 
independent of any other work. Thus $\mathrm{Br}=(\mathrm{IIO} .3 \mathrm{I} / 63.3727-\mathrm{I} .00000)$ 107.93 $=79.954$, a value almost identical with Baxter's value, 79.953. This is excellent proof that the bromine used in the present research was pure, and that the occlusion of electrolytes by silver bromide was small.

\section{The Atomic Weight of Potassium.}

The preceding paper and the present one together yield four ratios determined with modern precision, which together fix the atomic weight of potassium as definitely as could be expected. The respective values are as follows :

From the ratio of silver chloride to potassium chloride $\ldots \ldots \ldots \ldots \mathrm{K}=39.1 \mathrm{I} 34$ From the ratio of metallic silver to potassium chloride $\ldots \ldots \ldots \ldots \ldots \mathrm{K}=\mathbf{3 9} . \mathbf{1}$ 145 From the ratio of silver bromide to potassium bromide..........K =39.II35 From the ratio of metallic silver to potassium bromide.........K $=39$. II 43

Average atonic weight of potassium, if $\mathrm{Ag}=107.930 . \ldots \ldots \ldots \mathrm{K}=39.1 \times 39$

These figures are interesting and significant. The maximum departure from the mean is only I part in 70,000, and such differences as exist in the figures are explicable. It is likely that the slightly lower value given by the first member of each pair of series was due to a trace of occlusion of potassium nitrate by each of the precipitates - a circumstance which can not be absolutely prevented. Therefore the higher values, averaging 39. I I 44, are more probable. The differences are, however, wholly negligible at present.

In this connection it is interesting to note that Clarke in 1897 , from a miscellaneous collection of partly uncertain results obtained by others, decided upon the almost identical value, 39. I $2,{ }^{1}$ although at the same time from similar results he obtained values much too low for chlorine and iodine, somewhat too low for bromine, and much too high for sodium.

The very close mutual agreement of the new results obtained from two different compounds is a satisfactory verification of the relative values yielded by the recent work on the atomic weights of chlorine and bromine. ${ }^{2}$ The value of chlorine found from the work on potassium chloride was 35.475 ; and that of bromine from the present work is 79.954 . The ratio of chlorine to bromine is thus found to be 0.44369 , whereas the ratio computed from the work of Richards and Wells and of Baxter is 0.44367 .

Against such an accumulation of concordant data as that just presented, the older figures can have no important weight. Whatever may have been the cause of the irregularity and internal inconsistency of Stas's results with potassium chloride and bromide, there seems to be little reason

${ }^{1}$ Recalculations, p. 57.

${ }^{2}$ Richards and Wells, loc. cit.; Richards, Trans. Am. Phil. Soc., 43, II6 (Igor), Baxter, loc, cit. 
to doubt that the outcome of the present investigation, 39. I 4 , really represents the atomic weight of potassium.

It is needless to point out that this change in the atomic weight of potassium will affect many other atomic weights.

\section{Summary.}

This investigation upon the atomic weight of potassium presents, among other considerations, the following additions to the knowledge of the subject :

(I) The problem of preparing pure potassium bromide was solved in two ways.

(2) An unusually satisfactory method for preparing pure potassium hydroxide was developed. This method is applicable to other alkalies, and will be described elsewhere in greater detail.

(3) The ratio of silver to potassium bromide was redetermined, and found to be 100.000 : I10.3I9. The atomic weight of potassium was thus found to be 39.1143 , if silver is 107.930 and bromine 79.953 .

(4) The ratio of silver bromide to potassium bromide was found to be $100.000: 63.373$. This determination yielded an essentially equal value, $\mathrm{K}=39$. I I 35 .

(5) These values confirm in a striking manner the simultaneously executed work upon potassium chloride, and unite with them in showing that the atomic weight of potassium is 39 . I 4 .

(6) By thus agreeing, these four values support the new value for the atomic weight of chlorine in relation to silver and bromine.

\section{REACTIONS IN LIQUID AMMONIA.' (POTASSIUM AMMONOZINCATE, CUPROUS NITRIDE AND AN AMMONOBASIC MERCURIC BROMIDE)}

BY F. F. FITZGERALI).

Received January 20 , Iģo

Introduction.

The close relation between the properties of water and liquid ammonia has been pointed out by Prof. E. C. Franklin". Of all well known solvents ammonia resembles water most closely in its properties. As water is to be regarded as a compound of $\mathrm{H}$ and $\mathrm{OH}$ ions, so analogously ammonia is to be looked upon as a compound of $\mathrm{H}$ and $\mathrm{NH}_{2}$ ions. Acid amides and metallic amides are related to ammonia as the ordinary acids and bases are related to water. Franklin and Stafford have shown ${ }^{3}$ that acid amides and metallic amides react with each other to form a class of compounds which are related to ammonia as the ordinary oxygen salts are

1 From the anthor's thesis submitted to the Iepartment of Chemistry of the Leland Stanford Junior University for the Aegree of Masters of Arts.

'Am. Ch. J., 21, 8.

"Ibid., 28, 83 . 